\title{
Prediction of the First Tennis Serve Success Rate Based on Neural Network
}

\author{
Xi-Bin XU \\ College of Physical education, Xianyang Normal University, Xi'an, 71200, China \\ 23414388@qq.com
}

Keywords: Tennis, Serve, Neural Network, BP.

\begin{abstract}
The first serve success rate play very important position in tennis. In the thesis, we use the time sequence of tennis competition as the statistical data to predict the technical statistical indicators for the tennis player based on BP neural network algorithm. Our study aims at discussing the availability and applications of the BP neural network in the score prediction.
\end{abstract}

\section{Introduction}

The prediction of competition scores has increasingly become crucial in scientific sport managements and competitions. Also, it has been concerned by the leaders, managers and researchers during current days. With the development of dynamic system theories, artificial neural networks (ANNs) and expert system, sports predictors start to use better approaches that suits for human mind and the dynamic changes of predictive environments. ANNs can learn existing data automatically without too much complex processes and get close to the best function that can classify the samples [1]. Artificial expert system can gather the knowledge and experiences of human and give quantitative results, which makes the problems be more close to actual issues. Therefore, ANNs and artificial intelligence have successfully extend the potential development of predictive systems [2]. Here, we use the time sequence of tennis competition as the statistical data to predict the technical statistical indicators for the tennis player based on BP neural network algorithm. Our study aims at discussing the availability and applications of the BP neural network in the score prediction.

\section{Introduction to Artificial Neural Network System}

ANNs is a simulation of the neural networks of human brain, which mainly developed by simulating the neural structure and function of human brain[3]. It is made up of a large number of so-called "neurons" which interconnect with each other. Being able to operate the complex logical non-linear self-adaptive system, ANNs have a strong capacity to self-adapt, self-study, associative memorize and tolerate high false. It is suitable for dealing with complex information, incomplete data and the problems that are difficult to be described by mathematical models[4].

BP neural network is a kind of mature and widely-used ANN, which is made up of input, hidden and output layers. Units in different layers interconnect with each other and those in the same layer are independent. The basic principle of a BP neural network is: the information processing consists of the forward propagation and error back propagation. In terms of the forward propagation, information is inputted in the input layer, and then outputted by output layer after being processed by each hidden layer. If the outputted information in the output layer is not agreed with the desired information, the errors would be revised gradually. Because the BP neural network can precisely fit every continuous function, it has very good fitting effects in multi-factor and non-linear problems. Therefore, in this study, we use BP neural network to build the supplementary system for sport training.

\section{Principle of BP Neural Network}

1) Initialization, a certain reasonable structure of network is chosen and all the adjustable parameters (weights and thresholds) can be set as the minimal values evenly.

2) Calculate every sample as follows: 
1 Calculate forward, for the jth neuron of the lth layer, it holds that:

$$
v_{j}^{(l)}(n)=\sum_{i=0}^{T} \omega_{j i}^{(l)}(n) y_{i}^{(l-1)}(n)
$$

where $y_{i}^{(l-1)}$ (n) is the signal from the ith neuron of the former layer (l-1th layer) (when $i=0$, $\left.y_{0}^{(l-1)}(n)=-1, \omega_{j 0}^{(l)}(n)=\theta_{j}^{(l)}(n)\right)$. If the activated function of the jth neuron is the Sigmoid function, it holds that:

$$
\begin{aligned}
& y_{j}^{(l)}(n)=\frac{1}{1+\exp \left(-v_{j}^{(l)}(n)\right)} \text { and } \\
& \varphi\left(v_{j}(n)\right)=\frac{\partial y_{j}^{l}(n)}{\partial v_{j}(n)}=\frac{\exp \left(-v_{j}(n)\right)}{\left(1+\exp \left(-v_{j}(n)\right)^{2}\right.}=y_{j}(n)\left[1-y_{j}(n)\right]
\end{aligned}
$$

calculate $\delta$ oppositely

to the output unit it holds that:

$\delta_{j}^{l}(b)=e_{j}^{L}(n) o_{j}(n)\left[1-o_{j}(n)\right]$

to the hidden unit it holds that:

$$
\omega_{j k}^{(l)}(n+1)=\omega_{j i}^{(l)}+\eta \delta_{j}^{(l)}(n) y_{i}^{(l-1)}(n)
$$

revise the weights according to the equation shown as follows:

$$
\omega_{j k}^{(l)}(n+1)=\omega_{j i}^{(l)}+\eta \delta_{j}^{(l)}(n) y_{i}^{(l-1)}(n)
$$

3) when $n=n+1$, new sample (or new sample period) is inputted until $E_{A V}$ reach the defined requirements. Samples in different periods would be resequenced when training.

4) After training, the weights and thresholds have a defined function relationship between the input and output. Therefore, the network can start to work.

\section{Model Development for Predicting the Tennis Competition Scores using ANNs}

\section{Predictive Model Development Approaches of ANNs}

The ANN model for basketball competition scores is the method based on data. It means that using ANNs can generate a non-linear fitting process to get close to a time sequence or a similar time sequence. The future time values can be expressed utilizing the past time values via the clear logical relationship of ANNs.

Predictive ANNs can be divided into two kinds of predictions, the single-variable prediction based on time sequence and the multi-variables prediction based on time sequence. In terms of the single-variable prediction, we can set the time sequence as $\left\{X_{i}\right\}$, where $X_{n}, X_{n+1}, \cdots, X_{n+m}$. The prediction at the future $n+m+k(K>0)$ is the prediction of the value of $X_{n+m+k}$. That is to say, it draws 
the non-linear relationship of the historic data $X_{n}, X_{n+1}, \cdots, X_{n+m}$ and $X_{n+m+K}$. In terms of the multi-variables prediction, we set the time sequence as $\left(X_{1 n}, X_{2 n}, \cdots, X_{i n}\right),\left(X_{1 n+1}, X_{2 n+1}, \cdots\right.$, $\left.X_{i n+1}\right), \cdots,\left(X_{1 n+m}, X_{2 n+m}, \cdots, X_{i n+m}\right)$. We can predict the future values at $n+m+k(k>0)$. Being similar to the single-variable prediction, the ANNs can fit the non-linear relationship $X_{n+m+K}=F\left\{\left(X_{1 n}, X_{2 n}, \cdots, X_{i n}\right),\left(X_{1 n+1}, X_{2 n+1}, \cdots, X_{i n+1}\right) \cdots,\left(X_{1 n+m}, X_{2 n+m}, \cdots, X_{i n+m}\right)\right\}$.

Rolling prediction, the so-called iterated one-step prediction. We can do a single-step prediction, and give the feedback of output to the input layer, which can be used for predicting the values at $k$ kinds of times.

$\begin{array}{lcc}\text { Step } & \text { The neural network input } & \text { Output (prediction) } \\ 1 & X_{n}, X_{n+1}, \cdots, X_{n+m} & X_{n+m+1} \\ 2 & X_{n+1}, X_{n+2}, \cdots, X_{n+m+1} & X_{n+m+2} \\ \cdots & \cdots & \cdots \\ K & X_{n+k-1}, X_{n+k}, \cdots, X_{n+m-1} & X_{n+m+k}\end{array}$

\section{The Achievement of Rolling Prediction}

Here we take a technical statistical results of A tennis player to illustrate the approach of rolling prediction using ANNs. We collected five competition data in recent two years as the training sample. According to the existing mean the first serve success rate and the actual situations, we can set the first serve success rate of the input variable of the model. The statistical data is shown as follows (table 1):

Table 1 The first serve success rate a game

\begin{tabular}{ccccc}
\hline & 1 & 2 & 3 & 4 \\
\hline The first group & 0.7327 & 0.7226 & 0.7252 & 0.7082 \\
The second group & 0.7612 & 0.7116 & 0.6611 & 0.7638 \\
The third group & 0.6883 & 0.6515 & 0.7628 & 0.7327 \\
The fourth group & 0.7376 & 0.7106 & 0.7214 & 0.7594 \\
The fifth group & 0.7296 & 0.7905 & 0.7318 & 0.7257 \\
The Sixth group & 0.6915 & 0.6121 & 0.7459 & 0.7119 \\
\hline
\end{tabular}

To make the weights of networks are in the range of [0,1], we must do normalization of the inputted data, using the equation shown as follows:

$$
S_{i}=\frac{x_{i}-x_{\min }}{x_{\max }-x_{\min }}
$$

where $x_{\max }, x_{\min }$ are the maximum and minimum of original data respectively.

The processed data is shown as follows (table 2):

Table 2 The unitary data of serve a game

\begin{tabular}{ccccc}
\hline & 1 & 2 & 3 & 4 \\
\hline The first group & 0.7327 & 0.7226 & 0.7252 & 0.7082 \\
The second group & 0.7612 & 0.7116 & 0.6611 & 0.7638 \\
The third group & 0.6883 & 0.6515 & 0.7628 & 0.7327 \\
The fourth group & 0.7376 & 0.7106 & 0.7214 & 0.7594 \\
The fifth group & 0.7296 & 0.7905 & 0.7318 & 0.7257 \\
The Sixth group & 0.6915 & 0.6121 & 0.7459 & 0.7119 \\
\hline
\end{tabular}

\section{Design of BP network}

We use a three-layer BP network to predict the first serve success rate. There is one neuron in the input layer, 25 neurons in the hidden layer and 1 neuron in the output layer, which represents the predicted value of the first serve success rate. The activated functions of the hidden and output layers are Sigmoid and purelin functions respectively. Using the first four data in the table above it can 
predict the next data. Therefore, the input nodes are 4 and the output node is 1 . The function of nodes is the S-type function.

$$
f(x)=\frac{1}{1+e^{-x}}
$$

\section{Process of the Rolling Prediction Approach}

The rolling prediction of shooting percentage is the predictiong of the value in a future time based on a group of historic data. And then the predicted data will be seen as the historic data and make further prediction. In this cycle, the future values can be predicted step-by-step.

Network Training. We use the 1st statistical competition data as the input of the network, and the 2-5th technical statistical data is set as the ideal output of the network, consisting of the training sample to train the network until the strong generalization network occurs.

Network Prediction. We set the 1st data in the table as the input of the network. The 1st data in the 2nd group was outputted by the network. Then the entire output is set as the new input, acquiring the 2nd data in the 2nd group. Following this rolling regulation, we can predict that the normalized the first serve success rate of the player in the next stage of the competition is 0.8486 and the anti-normalized value is 0.6782 .

\section{Summary}

The two features of the BP neural network algorithm can help us resolve the problems and difficulties of uncertainty in sports. The first one is that BP neural network algorithm can learn and store plenty of input/output reflection relationship without knowing the mathematical equations. The second one is that BP neural network algorithm has a very good self-adaption and self-organization capacity. Therefore, BP neural network algorithm can be used as an approach for predicting the scores in sport competitions. Meanwhile, the use of neural network toolbox in Matlab can predict the scores conveniently, improving the efficiency and accuracy of the modeling process.

\section{References}

[1] Mal. R, Mal. D, K. Differentiating between good credits and bad credits using neuro-fuzzy systems, Artificial Intelligence and Information Technology. 136(2002) 190-211.

[2] J F. Wang, B Zhang, Study on New Adaptive Complex Equalizers Con-structde by Radial Basis Function Neural Networks, Systems Engineering and Electronics. 25(2003) 848-859.

[3] P F. Yan, C S. Zhang, Artificial neural network and simulated evolutionary computation, first ed., Tsinghua university press, Bei Jing, 2000.

[4] L M. Zhang, Artificial neural network model and its application, third ed., Fudan University Press, Shang Hai, 1999. 